metry please, hydramnios; and what constituted a positive radiological correlation in such cases? The method used for scoring agreements in this study (stated simply as "whether the radiological interpretation agreed with the clinical diagnosis as stated on the request card") is not clearly defined (except when nothing was detected on a routine -no pathology suspected-request). Perhaps the author had accurate and specific clinical diagnoses in all cases, but this is not stated or implied. The reverse is actually implied when he refers to such diagnoses as vertigo or headache.

I was, in fact, impressed with the overall detection rate of $6.3 \%$ additional (unsuspected) significant chest lesions discovered (differently stated by the author, it is even more impressive-"more than one-fifth of all lesion detected in the chest ... are not apparently suspected ... ") and almost $2 \%$ in the norma (routine) chests done. This is a high discovery rate in most present day surveys. Agreemen with a specific clinical diagnosis (pulmonar tuberculosis, intestinal obstruction, fetal mal formation, etc.) is quite different from radio logical detection on examinations done for symptoms or signs (cough, abdominal pain, hydramnios, etc.). In the former, clear agreement or disagreement can be recorded and the contribution towards disagreement by each party can be known when the true lesion is discovered. The use of radiology for detection purposes when there is no firm clinical or pathological diagnosis must be appraised differently. Perhaps this difference helps to explain the wide agreement difference of $1.4 \%$ for skulls (rarely is a true clinical diagnosis given) and $54 \%$ for barium meals. (Frequently, a true diagnosis is given.) Other factors are undoubtedly important also as the author states.

The author admits "this method has obvious limitations as there is no final pathological diagnosis". He then goes on to assum the initial clinical diagnosis (although headache or vertigo hardly seem to be diagnoses is the correct one, and considers the radiological diagnosis (such as no abnormality seen) wrong, if it does not agree, except when the clinician expected a normal result and the radiologist discovered a significant lesion This situation is simply counted as an agreement for the radiologist as it is added to his other agreements for his total score it is not counted as a disagreement for the clinician who suspected no pathology. True this method has the merit of simplicity he notes, but it has no scientific merit. To then suggest that a parallel study of follow-up request cards showed the initial clinical diagnosis was repeated (as yet unpublished by the author) supports the view that it was, therefore, likely to be the correct diagnosis, is to accept the words of the Bellman in the Hunting of the Snark-"What I tell you three times is true." (Lewis Carroll).

The author also notes that "it is not possible in the present series to measure the clinical contribution to the low agreement percentage" that is, no pathological confirmation was obtained. In a study of this nature, as previously suggested, pathological diagnosis is essential; if the clinical contribution to misdiagnosis cannot be determined, how can the radiological? (Of course, the reverse assumption could have been made, that the radiological diagnosis was the correct one and disagreement was due to the clinical misdiagnosis.)
I strongly agree with the author's final statement that it is extremely important for the student to be taught the applications of diagnostic radiology, but such teaching must be based on studies with more scientific validity than this one.-I am, etc.

Radiology Department
University of Ibadan, Ibadan, Nigeria S. P. BOHRER

\section{Experimental Infection of Man with} Pig Whipworm

SIR,-Trichuris suis, the pig whipworm has a worldwide distribution' as does the human whipworm Trichuris trichuira. Stoll estimated that there were 355 million in fected humans throughout the world. It is generally accepted that the two nematodes are distinct species. Dinnik" demonstrated minor morphological differences, including the sizes of eggs and infective larvae and in chromosome numbers; others have failed to differentiate between the worm types.' The differences reported, however, are such that they would not be detected by routine medical and veterinary parasitological techniques.

Recently, an experimental self-infection of Trichuris suis was achieved in man (aged 23 years). A single oral dose of 1,000 infective eggs suspended in $10 \mathrm{ml}$ of distilled water resulted in a patent infection detected by day 60 . Five stool samples, two taken prior to infection and three during the next 40 days were negative for Trichuris eggs, using the formalin-ether concentration technique. The eggs used were harvested from the faeces of three naturally infected sixmonth-old farm pigs and were cultured to the infective stage in moist vermiculite at $26^{\circ} \mathrm{C}$ for 34 days. Concurrently, four pigs, aged 9 weeks, were infected successfully with eggs from this same harvesting and culture; 30,000 eggs were given in a single dose by mouth and maturation of the parasites occurred between 41 and 49 days. In the human experimental infection no symptoms of distress and no diarrhoea were experienced, and no pronounced alterations in the differential white cell count could be detected. The number of eggs in the faeces has remained constant at 20 eggs per gramme in the ten week period after maturation occurred, and $11^{\circ}$ " have embryonated in culture indicating the presence of both male and female worms; the usua embryonation rate of $T$. suis eggs from pig faeces is $84-86 \%$.

A search of the literature has not yielded any previous reports of Trichuris suis experimental infections in man developing to maturation though Tukalevski, ${ }^{5}$ following a small dose of 87 infective eggs, isolated two larvae from his stool 19 days later. However, the dose was small and he discontinued stool examinations at 51 days.

The fact that this single human crossinfection was established with relative ease might suggest that the source of infection of some cases of trichuriasis in man could be the pig or its excreta, particularly in situations where man and pig have regular and close contact. It would be interesting to establish whether such an association exists. In the meantime, eggs from human cases of trichuriasis are being collected and processed in the hope of obtaining sufficient numbers to attempt human to pig Trichuris infection.-I am, etc.

R. J. S. BEER

Animal Health Trust,

Stock Livestock Research Centre,

1 Beer, R. J. S., Taffs, L. F., Jacobs, D. E., Lean, I. J., and Curran, $\dot{M}$. $\ddot{\mathbf{K}}$., Veterinary Record,

1971, in press.
3 Stoll, N. R., Journal of Parasitology, 1947, 33, 1. Dinnik, N. N.' Meditsinskaya Parazitologiya Parazitarnye Bolezni, 1938, 7, 907. Research, 1926, 33, 31.

kalevski, I. M. Meditsinskya Purazio
Parazitarnye Bolezni, 1940, 9, 444.

Recurrent Erythema Nodosum of Pregnancy

SIR,-Erythema nodosum is probably a hypersensitivity reaction characterized by inflammatory nodules in the dermis and subcutaneous tissue. It is regarded as secondary to a variety of underlying disorders, especially bacterial, fungals and viral infections and drug reactions, but may occur in pregnancy with no underlying cause found, as in the following case.

The patient's first pregnancy at 18 years of age was complicated by a rash of red, tender nodules over both her shins. This rash first appeared at 32 weeks amenorrhoea and fresh crops of nodules appeared regularly up to the time of delivery. After the normal delivery at term of a male infant weighing $3.5 \mathrm{~kg}$, the rash began to fade and had disappeared completely by the end of the second week. Full investigation at the time failed to show an underlying cause.

One year later the patient's second pregnancy was similarly complicated by the same type of rash, over the same area, again from the 32 week onwards and fading after delivery of a male infant weighing $4.0 \mathrm{~kg}$ In each pregnancy the patient obtained the best relief by wearing elastic web bandages. These would appear to control the oedema around the nodules. No analgesia or corticosteroid treatment was required. I am, etc.,

Nether Fuge Hospital,

E. DAw

Shefficlu, Yorks.

\section{Pyrexia and Dialysis}

SIR,-The experience of Drs. P. J. A. Robinson and S. M. Rosen (6 March, p. 528) was shared by the Auckland Dialysis Unit in 1968. Over a four-month period 18 patients in a six-bed dialysis unit had 116 episodes of hyperpyrexia with rigors and hypotension in 519 dialyses. Several patients required hospital admission for intensive treatment.

A Biosystems MAKS machine supplied dialysate from a tank delivery system to Kiil dialysers. The tanks and lines were cleaned with Chlornox and formaldehyde 2"'.. The Kiil dialysers were built with wet cuprophan membranes, sterilized with $2 \%$ formaldehyde for six hours, drained, then flushed with two litres heparinized saline in the blood compartment, and tap water at $4 \mathrm{lb} /$ in $^{2}\left(0.28 \mathrm{~kg} / \mathrm{cm}^{2}\right)$ for 30 minutes in the dialysate compartment. The dialysers were not shared between patients, new sterile blood lines were used each dialysis, and bacterial counts and culture samples were taken routinely from the emergent saline as the patient was connected to the dialyser. 